\title{
Performance computation of cross-layer Hybrid ARQ schemes at IP layer in the presence of corrupted acknowledgments
}

\author{
Sébastien Marcille*† \\ sebastien.marcille@fr.thalesgroup.com \\ sebastien.marcille@telecom-paristech.fr
}

\author{
Philippe Ciblat* \\ *TELECOM PARISTECH \\ 75013 Paris, France \\ philippe.ciblat@telecom-paritech.fr
}

\author{
Christophe J. Le Martret ${ }^{\dagger}$ \\ $\dagger_{\text {THALES Communications and Security }}$ \\ 92704 Colombes CEDEX, France \\ christophe.le_martret@fr.thalesgroup.com
}

\begin{abstract}
Hybrid ARQ (HARQ) schemes operate at the PHY and Medium Access (MAC) layers and their performance have been naturally studied at the MAC level. However, all the modern systems are going to be running under the IP protocol. Therefore, in order to get realistic performance of the whole system considering the multiple layer stacks, performance analysis at the IP layer is crucial. Very little work has been done so far in this direction, except in [2] which studies the delay statistics of IP packets with Selective-Repeat ARQ, and [1] which proposes a cross-layer optimization strategy between MAC and IP layers for $A R Q$, and in [8], [9] which consider the HARQ case. In this paper, we study the effect of erroneous feedbacks at the MAC layer on the performance at the IP layer considering both conventional HARQ schemes as well as the cross-layer strategy mentioned above. We derive in closed-form expressions the performance in terms of packet error rate (PER), efficiency, and delay with respect to the error probability of the feedback channel.
\end{abstract}

\section{INTRODUCTION}

HARQ protocols combine ARQ retransmission schemes along with channel coding capabilities. They enable to provide reliable link transmission in wireless systems in varying channels. They have been used from the beginning in cellular standards (GSM GPRS/EDGE) and are still included in the most recent ones (WiMAX, 3GPP LTE). The motivation of this work is twofold. First, we conduct the performance study at the IP layer. Although the design of conventional HARQ system is done at the Medium Access Control (MAC) layer, most of the systems run an IP protocol and it is thus of great importance to study the performance at the IP layer. Indeed, in the spirit of the cross-layer concept, the optimum performance at the MAC layer does not guarantee that the performance will be optimal at the IP layer. Moreover, a recent crosslayer strategy between the MAC layer and the IP layer has been proposed in [1] in order to enhance the performance at the IP layer and thus for which the performance at the MAC does not make sense. Second, retransmission schemes are implemented using an acknowledgment process sent by the receiver which tells the transmitter whether the packets are correctly received (ACK) or not (NACK). A lot of work has been done investigating HARQ performance under the assumption of error-free (referred to as perfect in the sequel) feedback. Analytical studies are found, for instance [3]-[7] focused on MAC level, [8]-[10] on network level and [11] on application level. However, since the acknowledgments are transmitted over the air in wireless systems, they are prone to transmission errors. Only a small amount of analytical studies about the impact of non-perfect feedback on HARQ have been done and only at MAC layer. Some works studied the efficiency of Type-I HARQ in the case of an infinite number of retransmissions [12], [13]. In the case of finite retransmissions, analytical expressions of the efficiency were derived in [14] for a Type-I HARQ and in [15] for the Type-II HARQ with Chase Combining. All the previously mentioned references tackle the Stop and Wait (SW) protocol. Other analysis have been done based on Markov chains for the Selective Repeat (SR) protocol [16]-[18] and for the Go-Back-N (GBN) protocol [19]. Thus, our goal in this paper is to derive in closed-form the Packet Error Rate (PER), efficiency and delay at the IP layer when the feedback (fb) channel is corrupted. Moreover we will consider both conventional and cross-layer strategy. Note that our results are an extension of [8], [9] to the case of corrupted feedback.

The paper is organized as follows. We first describe the system model in Section II including the corrupted feedback channel and the cross-layer strategy. Then, we derive the performance metric in closed-form in Section III starting with the cross-layer strategy, the conventional one being deduced as a particular case. Section IV is devoted to numerical illustrations. Concluding remarks are drawn in Section V.

\section{SYSTEM MODEL}

\section{A. Layer and HARQ model}

We focus on the first three layers of the ISO model respectively referred in the sequel to as PHY (physical layer - layer 1), MAC (medium access layer - layer 2) and NET (network layer - layer 3). We assume that the NET protocol is the Internet Protocol (IP) but our work can be applied to any other protocol. We also assume that the incoming IP datagrams are fragmented at the MAC layer into $N$ fragments (FR) of equal length. Each fragment is then transmitted following an HARQ process, i.e. transformed into MAC packet(s) according to the considered HARQ scheme and then encoded and sent 
through the wireless channel. At the receiver side, the PHY packet is decoded and sent to the MAC layer. The HARQ process then tries to decode without error the fragment using the new received MAC packet, and sends back to the transmitter an acknowledgment message (ACK or NACK) accordingly. If the transmitter receives ACK, it starts the HARQ process with the next fragment. Otherwise, it sends another MAC packet following the considered type of HARQ. Once the $N$ fragments are correctly received, the receiver reassembles it into an IP datagram that is sent to the NET layer. The number of trials per fragment is usually limited to bound the maximum transmission delay, and we denote with $M$ the maximum number of transmissions (also referred in the sequel as credit) allowed for a single fragment. Note that the assumptions made in this work follow those of [8], [9]: $i)$ the channel realizations are independent between two successive transmissions (symbol or packet), ii) we assume equal length for all the MAC packets (fits a large amount of HARQ schemes).

\section{B. Cross-layer strategy}

The conventional retransmission schemes depicted in the previous section are usually implemented at MAC layer where the fragments are managed independently one after the other. For truncated schemes, if the $M$-th transmission of a same fragment fails, this fragment is dropped and the retransmission process is restarted with the next fragment. Recently, based on the fact that an IP packet is dropped by the NET layer if at least one of its fragment is missing at the receiver side, authors of [1] proposed to enhance the ARQ scheme by granting a global transmission credit, denoted $C$, to the set of fragments belonging to the same IP packet before being reassembled. Thus, rather than allowing each of the $N$ fragments to be transmitted $M$ times, the new scheme allocates $C$ transmissions to this set of $N$ fragments. Results in [1] show that this cross-layer strategy outperforms the conventional one in terms of PER. In the later we will refer to the conventional strategy as Fragment-Based Strategy (FBS) and to the crosslayer one as XL-Based Strategy (XBS). As shown in [8], this strategy can be applied to any type of HARQ.

\section{Feedback channel model}

We assume that the acknowledgments are encoded with a CRC that is strong enough in order to neglect the probability of misdetection. Thus, each time the acknowledgment is corrupted (contains at least one error), the CRC detects it. In that case the acknowledgment will systematically be interpreted as a NACK. With this protocole, a corrupted NACK will be received as a NACK (no influence) and a corrupted ACK will be received as a NACK too. Indeed, taking a NACK instead of an ACK is much less damageable than the contrary. Finally, the event $\{\mathrm{ACK} \rightarrow \mathrm{NACK}\}$ occurs with probability denoted $p_{\mathrm{fb}}:=\operatorname{Pr}\{$ an acknowledgment is corrupted $\}$.

\section{Metrics definitions}

In this paper we conduct the analysis of the three following metrics: the average packet error rate, the efficiency, and the average packet delay. In FBS, the metrics can be computed both at MAC level (where the fragments are the unit of interest) and at IP level (where the IP packets are the unit of interest). While in XBS, the metrics are defined at IP level only.

The packet error rate, denoted with $P$, is defined as the probability that a packet transmission fails. The efficiency is denoted with $\eta$, and is defined as the average number of correctly received bits per transmitted bit. Finally the packet delay, denoted with $n$, is defined as the average number of MAC packets needed to receive a single fragment (or IP datagram) without error.

For notation convenience, a subscript ' $F R$ ' (resp. ' $I P$ ') will stand for the MAC (resp. IP) level. A superscript ' $F$ ' will stand for the conventional strategy FBS, and ' $X$ ' for the cross-layer strategy XBS. Notice that a tilde $(\sim)$ will stand over those metrics evaluated under the corrupted feedback assumption.

\section{Performance derivation at IP LAYER}

\section{A. Cross-layer strategy}

Within this cross-layer context, the packet error probability depends on the quality of the feedback channel. Indeed, the remaining number of transmissions for the $i$ th fragment is driven by the number of transmissions consumed by the $(i-1)$ previous fragments. If the previous fragments wasted some transmissions due to some corrupted ACK, the fragment $i$ will less probably succeed since less transmissions will be allocated to it. Let us denote with $\tilde{p}_{n}(i)$ the probability of decoding $n$ fragments in $i$ transmissions and receiving $n$ ACKs (at the transmitter side).

Let us focus on the IP packet success probability. A packet will be correctly received iff the $N$ fragments are correctly received in $i$ transmissions with $i \in\{N, \ldots, C\} . N$ fragments need $i$ transmissions for correct reception if, for each $k \in$ $\{N-1, \ldots, i-1\}$,

i) the first $N-1$ fragments are acknowledged (and thus "sent" which means that the corresponding ACKs have effectively been received at the transmitter side), in $k$ transmissions.

ii) Next, the last $N$ th fragment is sent and correctly received in $(i-k)$ transmissions (regardless of the ACK/NACK value received at the transmitter side).

The event i) occurs with probability $\tilde{p}_{N-1}(k)$ and the event ii) with probability $p_{1}(i-k)$. Therefore, we obtain

$$
\tilde{P}_{\mathrm{IP}}^{\mathrm{X}}=1-\sum_{i=N}^{C} \sum_{k=N-1}^{i-1} \tilde{p}_{N-1}(k) p_{1}(i-k) .
$$

Using similar arguments than [8], it is found

$$
\tilde{n}_{\mathrm{IP}}^{\mathrm{X}}=\frac{1}{1-\tilde{P}_{\mathrm{IP}}^{\mathrm{X}}} \sum_{i=N}^{C} i \frac{\tilde{p}_{N}(i)}{\left(1-p_{\mathrm{fb}}\right)^{\mathbf{1}\{i=C\}}},
$$

where $1\{A\}$ is the Kronecker symbol of event $A$. The term $\left(1-p_{\mathrm{fb}}\right)^{1\{i=C\}}$ appears in order to take into account for the last ACK (relative to the last fragment) needs not be 
necessarily received when the whole transmission credit $C$ has been reached (i.e., when no transmission can further take place).

We recall that $\tilde{n}_{\mathrm{IP}}^{\mathrm{X}}$ is the average number of MAC packets needed to receive one IP packet (i.e. $N$ successive fragments) under imperfect feedback channel. Efficiency is given by

$$
\tilde{\eta}_{\mathrm{IP}}^{\mathrm{X}}=\frac{\rho N\left(1-\tilde{P}_{\mathrm{IP}}^{\mathrm{X}}\right)}{C \tilde{P}_{\mathrm{IP}}^{\mathrm{X}}+\left(1-\tilde{P}_{\mathrm{IP}}^{\mathrm{X}}\right) \tilde{n}_{\mathrm{IP}}^{\mathrm{X}}},
$$

where $\rho$ is the ratio between the fragment length and the PHY packet length.

Under XBS, the three metrics are determined by the knowledge of $p_{1}(i)$ and $\tilde{p}_{n}(i)$. We remind that closed-form expressions for $p_{1}(i)$ are available in [8]. The probability $\tilde{p}_{n}(i)$ is given in the next proposition which is one of the main contributions of this paper.

Proposition 1: $\forall n \geq 1$, the probability

$$
\tilde{p}_{n}(i)=\left(1-p_{\mathrm{fb}}\right)^{n} \sum_{q \in Q_{i, n}} \prod_{j=1}^{n} \sum_{k_{j}=1}^{q_{j}} p_{1}\left(k_{j}\right) p_{\mathrm{fb}}{ }^{q_{j}-k_{j}},
$$

with

$$
Q_{i, n}=\left\{\underline{q} \in \mathbb{N}_{*}^{n} / \sum_{j=1}^{n} q_{j}=i\right\} .
$$

Proof: For $i \geq n$, we obtain by direct enumeration

$$
\begin{gathered}
\tilde{p}_{n}(i)=\sum_{\underline{q} \in Q_{i, n}} \prod_{j=1}^{n} \operatorname{Pr}\left\{\mathrm{FR} \# j \text { received in } q_{j},\right. \\
\text { and ACK received }\} .
\end{gathered}
$$

The event $\left\{\mathrm{FR} \# j\right.$ received in $q_{j}$ and ACK received $\}$ is splitted as follows: $k_{j}$ transmissions until correct decoding of the fragment (at the receiver side), next $\left(q_{j}-k_{j}\right)$ transmissions for correct ACK reception at the transmitter side. This leads to

$$
\begin{aligned}
& \operatorname{Pr}\left\{\mathrm{FR} \# j \text { received in } q_{j} \text { and ACK received }\right\} \\
= & \sum_{k_{j}=1}^{q_{j}} p_{1}\left(k_{j}\right) p_{\mathrm{fb}}{ }^{q_{j}-k_{j}}\left(1-p_{\mathrm{fb}}\right) .
\end{aligned}
$$

For the set $Q_{i, n}$ becomes huge for practical values of $i$ and $n$, direct application of (4) for $\tilde{p}_{n}(i)$ is difficult in practice. This drawback is circumvented by remarking that this term can be calculated recursively as stated in the next proposition.

Proposition 2: The following recursion holds:

$$
\begin{aligned}
& \tilde{p}_{1}(i)=\left(1-p_{\mathrm{fb}}\right) \sum_{m=1}^{i} p_{1}(m) p_{\mathrm{fb}}{ }^{i-m}, \\
& \tilde{p}_{n}(i)=\sum_{j=1}^{i-n+1} \tilde{p}_{n-1}(i-j) \tilde{p}_{1}(j), \quad \forall n \geq 2 .
\end{aligned}
$$

Proof: $Q_{i, n}$ may be partitioned as follows: $n$ fragments are received in $i$ transmissions iff the $(n-1)$ previous fragments are received in $(i-j)$ trials, for $j \in\{1, \ldots, i-n+1\}$.
The procedure initialisation is given by (5), the probability of receiving one fragment with $C$ maximum transmissions.

\section{B. Conventional strategy}

Under the conventional strategy FBS, the packet error probability is not modified when the feedback channel is imperfect. The fact that ACK can be modified into NACK through the reverse link, and hence that some useless retransmissions may hold, does not change the good decoding of fragments at the receiver side. As a result, $\tilde{P}_{\mathrm{IP}}^{\mathrm{F}}=P_{\mathrm{IP}}^{\mathrm{F}}$ as it was already mentioned in [15] (which focused on MAC level only). We remind that closed-form expressions for $P_{\mathrm{IP}}^{\mathrm{F}}$ are available in [8].

Nevertheless, the fragments that are sent after reception of a corrupted ACK lead to a loss in efficiency and delay at MAC and IP level since, for instance, the transmitter will send useless redundant fragments whereas it should have sent new data fragments if the ACK were correct. The reader may notice that the average number of MAC packets that have been sent when the fragment is not correctly received is identical to the perfect feedback case. Hence, by following the same rationale as in [8], it is easily found, under i.i.d. fragments assumption, that

$$
\tilde{n}_{\mathrm{IP}}^{\mathrm{F}}=N \tilde{n}_{\mathrm{FR}}
$$

and

$$
\tilde{\eta}_{\mathrm{IP}}^{\mathrm{F}}=\frac{\rho\left(1-P_{\mathrm{FR}}\right)^{N}}{M P_{\mathrm{FR}}+\left(1-P_{\mathrm{FR}}\right) \tilde{n}_{\mathrm{FR}}},
$$

where $\tilde{n}_{\mathrm{FR}}$ is the average number of MAC packet needed to receive a single fragment without error (work is thus done at MAC level), with imperfect feedback. The term $P_{\mathrm{FR}}$ is the fragment error probability (MAC level too). Thus, the delay $\tilde{n}_{\mathrm{FR}}$ only has to be evaluated in closed-form. It can be obtained from Eq. (2) with $n=1$ and with $P_{\mathrm{FR}}$ instead of $\tilde{P}_{\mathrm{IP}}^{\mathrm{X}}$. We find

$$
\tilde{n}_{\mathrm{FR}}=\frac{1}{1-P_{\mathrm{FR}}} \sum_{i=1}^{M} i \frac{\tilde{p}_{1}(i)}{\left(1-p_{\mathrm{fb}}\right)^{\mathbf{1}\{i=M\}}},
$$

with $\tilde{p}_{1}(i)$ given by Eq. (5).

\section{Comparison with the literature}

We would like to compare our results with the literature. First of all, perfect feedback results given in [8], [9] are retrieved by setting $p_{\mathrm{fb}}=0$ in all the previous equations. To our best knowledge, all the derivations given in the present paper are new for IP layer whith imperfect feedback. Thus, in order to do the comparison with the state-of-the-art work involving imperfect feedback, we have to focus on MAC level and therefore have to set $N=1$ in our proposed expressions.

1) The efficiency is the same as that provided in [15]. The delay given in Eq. (9) is different from that given in [15, Eq. (6)]: the delay defined by the authors of that work is the efficiency inverse, which matches our definition for infinite maximum transmission credit only. 
2) For Type-I HARQ, we have $p_{1}(i)=\left(1-\pi_{0}\right) \pi_{0}{ }^{i-1}$ with $\pi_{0}:=\operatorname{Pr}\{$ MAC packet KO $\}$. Then, the delay expression can be dramatically simplified as follows

$$
\begin{aligned}
\tilde{n}_{\mathrm{FR}} & =\frac{1-\pi_{0}}{\left(1-P_{\mathrm{FR}}\right)\left(p_{\mathrm{fb}}-\pi_{0}\right)}\left(M\left(p_{\mathrm{fb}}{ }^{M}-\pi_{0}{ }^{M}\right)\right. \\
& \left.+\left(1-p_{\mathrm{fb}}\right)\left(p_{\mathrm{fb}} f_{M}\left(p_{\mathrm{fb}}\right)-\pi_{0} f_{M}\left(\pi_{0}\right)\right)\right),
\end{aligned}
$$

with $f_{n}(x):=\sum_{k=1}^{n-1} k x^{k-1}$. For infinite transmission credit, we have

$$
\lim _{M \rightarrow \infty} \tilde{n}_{\mathrm{FR}}=\frac{1}{1-\pi_{0}}+\frac{p_{\mathrm{fb}}}{1-p_{\mathrm{fb}}}
$$

which is in perfect agreement with that given in [12], [13] and [14, Eq. (17)].

As a conclusion, the new closed-form expressions for delay and efficiency at IP level only depend on the new computed delay at MAC level in imperfect feedback context. Our expressions (at MAC and IP levels) are general since they hold for any HARQ type and transmission credit.

\section{NUMERICAL ILLUSTRATIONS}

The results will be illustrated using two different HARQ types:

i) a pure ARQ scheme (corresponding to a Type-I HARQ without coding),

ii) and a Type-II HARQ scheme (basically, a scheme using the so-called Chase combining at the receiver, which is denoted CC-HARQ in the sequel).

Both FBS and XBS strategies are evaluated. The simulations are done over. Each MAC packet contains 128 information bits. A $1 / 2$ rate convolutional code with generator polynomials $(35,23)$ is used in CC-HARQ. A QPSK modulation/demodulation and an additive Gaussian (AWGN) channel mainly constitute the PHY layer.

In Fig. 1, we plot the theoretical (using (3) and (8)) and empirical (through Monte-Carlo simulation) efficiencies versus signal-to-noise ratio (SNR), with the feedback channel error probability fixed to $p_{\mathrm{fb}}=10^{-1}$. The analytical expressions are in perfect agreement with the simulations which confirms the accuracy of our derivations.

In Fig. 2, the delay is displayed for ARQ and CC-HARQ under perfect and imperfect feedback channels. In the following we describe how the reverse link was implemented. When the feedback channel is imperfect, the ACK/NACKs are inserted into packets of 32 bits ( 1 bit for ACK/NACK, 15 bits may contain other information such as the packet number or channel state information, and 16 bits CRC in order to detect errors in the 16 feedback informative bits). These 32 bits may be encoded (referred to as "coded fb") through the convolutional code used for the direct link, or not (referred to as "uncoded $\mathrm{fb}$ "). Finally the reverse link is assumed to be AWGN with the same SNR as the direct link.

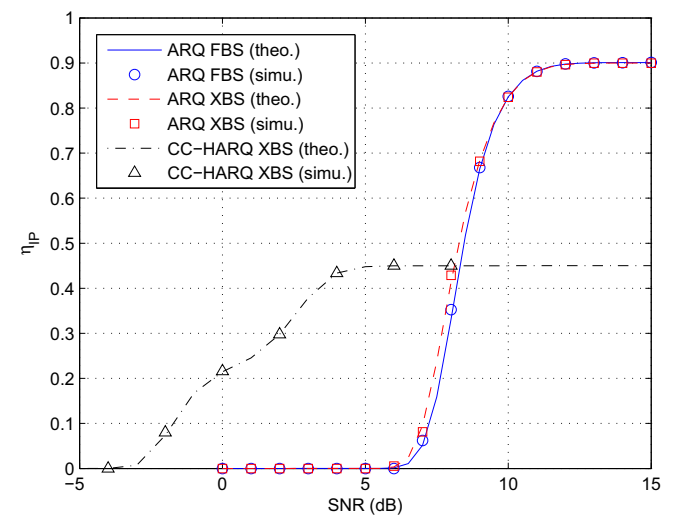

Fig. 1. Theoretical and empirical efficiency (IP) versus $\operatorname{SNR}(N=3$, $M=3, C=9$ ).

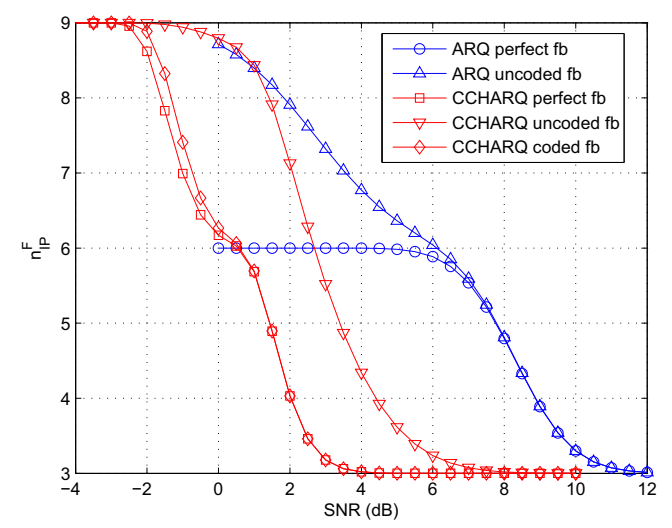

Fig. 2. Delay (IP) for ARQ and CC-HARQ under various feedback schemes (FBS, $N=3, M=3$ ).

We observe that for both schemes the delay is dramatically influenced by the feedback channel quality, and hence the feedback information has to be well protected against the feedback channel in order to approach the same performance as with perfect feedback.

In Fig. 3, the efficiency is plotted versus SNR for CCHARQ (in FBS and XBS contexts) for perfect feedback and imperfect feedback (with or without coding) modeled as AWGN. We observe that XBS is far more sensitive to imperfect feedback than FBS. It highlights the fact that the cross-layer design of HARQ schemes must be done with great care. Once again, it is shown that some overhead must be added in order to approach perfect feedback performance, especially in the cross-layer design.

It is more evidenced on Fig. 4, where we plot the PER versus SNR for CC-HARQ (in FBS and XBS contexts) with different feedback managements: perfect, or assumed to have fixed error probability $p_{\mathrm{fb}}$, or uncoded AWGN, or coded 


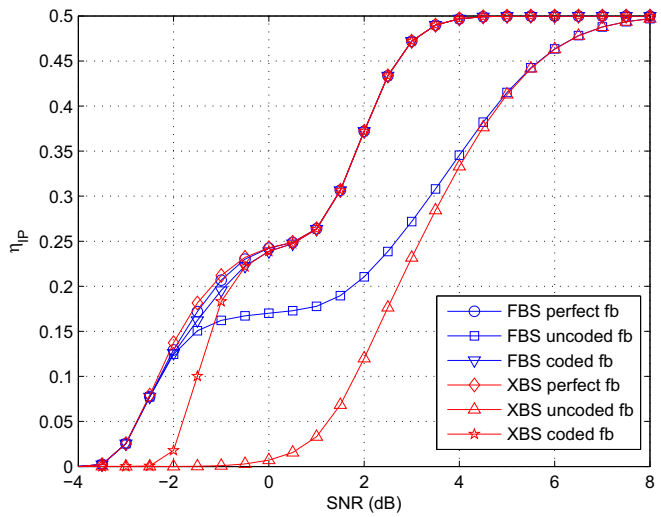

Fig. 3. Efficiency (IP) for CC-HARQ under various feedback schemes $(N=$ $3, M=3, C=9)$.

\section{AWGN.}

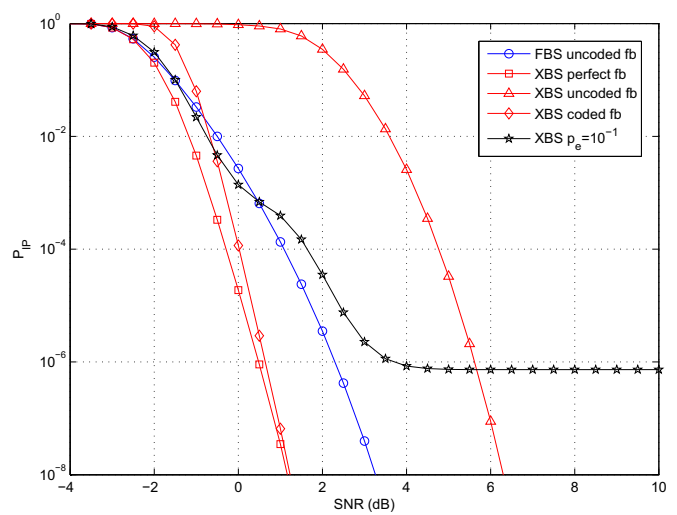

Fig. 4. PER (IP) for HARQ under various feedback schemes $(N=3$, $M=3, C=9$ ).

It is shown, as expected, that the PER of FBS is not sensitive to any imperfect feedback. In contrast, the PER of XBS may be far away from the ideal case. As the main advantage of XBS is to improve the PER [1], the feedback channel has to be well designed for XBS in order to still have a practical interest.

\section{CONCLUSION}

The impact of an imperfect feedback channel introducing some noise in the feedback information (corrupted ACK) has been analyzed on the performance of any HARQ scheme. The performance have been evaluated through three metrics of interest: the PER, the delay and the efficiency. The derivations have been made at IP layer, under two retransmission managements: the conventional fragmented approach (between MAC and IP layers) as well as a cross-layer design that originally improves the PER. The numerical illustrations reveal that the feedback channel has a non negligible influence on the performance, especially for the cross-layer designed scheme.

\section{REFERENCES}

[1] Y. Choi, S. Choi, and S. Yoon, "MSDU-based ARQ scheme for IP-level performance maximization," in Global Telecommunications Conference (GLOBECOM), vol. 5. IEEE, Oct. 2005, pp. 2495-2499.

[2] M. Rossi and M. Zorzi, "Analysis and heuristics for the characterization of Selective Repeat ARQ delay statistics over wireless channels", in IEEE Trans. Veh. Technol., vol. 52, no. 5, pp. 1365-1377, Sep. 2003.

[3] C. Lott, O. Milenkovic, and E. Soljanin, "Hybrid ARQ: theory, state of the art and future directions," in International Workshop on Information Theory (ITW). IEEE, Jul. 2007.

[4] S. Kallel, "Analysis of a type II Hybrid ARQ scheme with code combining," IEEE Trans. Commun., vol. 38, no. 8, pp. 1133-1137, Aug. 1990.

[5] E. Soljanin, R. Liu, and P. Spasojevic̀, "Hybrid ARQ with random transmission assignments," in Advances in network information theory: DIMACS Workshop, Network Information Theory, March 17-19, 2003, Piscataway, New Jersey. AMS Bookstore, 2004, p. 321.

[6] Q. Chen and P. Fan, "Performance analysis of Hybrid ARQ with code combining over interleaved Rayleigh fading channel," IEEE Trans. Veh. Technol., vol. 54, no. 3, pp. 1207-1214, May 2005.

[7] M. Levorato and M. Zorzi, "Performance analysis of type II Hybrid ARQ with Low-Density Parity-Check codes," in International Symposium on Communications, Control and Signal Processing (ISCCSP), 2008, pp. 804-809.

[8] A. Le Duc, C. J. Le Martret, and P. Ciblat, "Packet error rate and efficiency closed-form expressions for cross-layer hybrid ARQ schemes," in Signal Processing and Wireless Communications (SPAWC). IEEE, 2009.

[9] A. Le Duc, C. J. Le Martret, and P. Ciblat, "Delay and Jitter closed-form expressions for cross-layer hybrid ARQ schemes," in Proc. of the IEEE International Vehicular Technology Conference (VTC) Fall, (Anchorage, AL, USA), pp. 2266-2270, Sept. 2009.

[10] A. Le Duc, C. J. Le Martret, and P. Ciblat, "Efficiency closed-form expressions for any IR-HARQ scheme at the IP level," in Signal Processing and Wireless Communications (SPAWC). IEEE, 2010.

[11] I. Andriyanova and E. Soljanin, "IR-HARQ schemes with finite-length punctured LDPC codes over the BEC," in International Workshop on Information Theory (ITW). IEEE, 2009.

[12] W. El bahri, H. Boujemâa, and M. Siala, "Effects of noisy feedback on the performance of HARQ schemes over multipath block fading channels for DS-SSS," in 16th International Symposium on Indoor, Personal and Mobile Radio Communications. IEEE, 2005, pp. 25522556.

[13] S. B. Wicker, Error Control Systems for Digital Communications and Data Storage. Englewood Cliffs, NJ: Prentice-Hall, 1995.

[14] P. Wu and N. Jindal, "Coding versus ARQ in fading channels: how reliable should the PHY be?" Global Telecommunications Conference (GLOBECOM), 2009.

[15] E. Malkamäki and H. Leib, "Performance of truncated type-II Hybrid ARQ schemes with noisy feedback over block fading channels," IEEE Trans. Commun., vol. 48, no. 9, pp. 1477-1487, Sep. 2000.

[16] M. Rossi, L. Badia, and M. Zorzi, "SR ARQ delay statistics on Nstate Markov channels with non-instantaneous feedback," IEEE Trans. Wireless Commun., vol. 5, no. 6, pp. 1526-1536, Jun. 2006.

[17] K. Ausavapattanakun and A. Nosratinia, "Analysis of selective-repeat ARQ via matrix signal-flow graphs," IEEE Trans. Commun., vol. 55, no. 1, pp. 198-204, Jan. 2007.

[18] L. Badia, "On the effect of feedback errors in Markov models for SR ARQ packet delays," in Global Telecommunications Conference (GLOBECOM). IEEE, 2009.

[19] K. Ausavapattanakun and A. Nosratinia, "Analysis of go-back-N ARQ in block fading channels," IEEE Trans. Wireless Commun., vol. 6, no. 8, pp. 2793-2797, Aug. 2007. 